\title{
Efficacy of Ginger Supplementation in Relieving Persistent Hypothyroid Symptoms in Patients with Controlled Primary Hypothyroidism: A Pilot Randomized, Double-Blind, Placebo-Controlled Clinical Trial
}

\author{
Hamide Ashraf $\left(\mathbb{D},{ }^{1,2}\right.$ Mojtaba Heydari $(\mathbb{D})^{2}$ Mesbah Shams $\mathbb{D}{ }^{3}$, \\ Mohammad Mehdi Zarshenas $\mathbb{D}^{\mathbb{4},{ }^{4,5,6}}$ Ali Tavakoli $\mathbb{D}^{1,2}$ and Mehrab Sayadi $\mathbb{D}^{7}$ \\ ${ }^{1}$ Department of Persian Medicine, School of Medicine, Shiraz University of Medical Science, Shiraz, Iran \\ ${ }^{2}$ Research Center for Traditional Medicine and History of Medicine, Shiraz University of Medical Sciences, Shiraz, Iran \\ ${ }^{3}$ Endocrinology and Metabolism Research Center, Shiraz University of Medical Science, Shiraz, Iran \\ ${ }^{4}$ Medicinal Plants Processing Research Center, School of Pharmacy, Shiraz University of Medical Science, Shiraz, Iran \\ ${ }^{5}$ Department of Phytopharmaceuticals (Traditional Pharmacy), School of Pharmacy, Shiraz University of Medical Science, \\ Shiraz, Iran \\ ${ }^{6}$ Epilepsy Research Center, Shiraz University of Medical Sciences, Shiraz, Iran \\ ${ }^{7}$ Cardiovascular Research Center, Shiraz University of Medical Sciences, Shiraz, Iran
}

Correspondence should be addressed to Mesbah Shams; shams@sums.ac.ir and Ali Tavakoli; tavakkolia@sums.ac.ir

Received 15 July 2021; Revised 31 December 2021; Accepted 4 January 2022; Published 20 January 2022

Academic Editor: Gianluca Tamagno

Copyright $(92022$ Hamide Ashraf et al. This is an open access article distributed under the Creative Commons Attribution License, which permits unrestricted use, distribution, and reproduction in any medium, provided the original work is properly cited.

Primary hypothyroidism is a common disease. Some patients have persistent symptoms despite normal serum thyroid-stimulating hormone (TSH) levels. Ginger is reported to be beneficial in relieving similar symptoms. Our aim was to evaluate the efficacy of ginger supplementation in relieving persistent symptoms in these patients. In this randomized, double-blind, placebocontrolled clinical trial, 60 hypothyroid patients aged 20-60 years with normal serum TSH concentrations were randomly allocated to two equal parallel study groups of ginger ( $500 \mathrm{mg}$ twice a day) or placebo for 30 days. Hypothyroid symptoms were evaluated as the primary outcome using the Thyroid Symptom Rating Questionnaire (ThySRQ) before and after the intervention. Anthropometric measures and laboratory indices including TSH, triglyceride (TG), total cholesterol (TChol), and fasting blood sugar (FBS) were considered as secondary outcomes. A significant lower mean total ThySRQ score $(8.63 \pm 5.47 \mathrm{vs} .15 .76 \pm 6.09$, $P<0.001)$ was observed in the ginger group compared to the control group. Ginger led to significant improvements in the mean scores of the weight gain, cold intolerance, constipation, dry skin, appetite, memory loss, concentration disturbance, and feeling giddy or dizzy domains $(P<0.001)$. However, no significant improvements were observed in hair loss, nail fragility, hearing, hoarseness, speech, and depression or feeling down $(P>0.05)$. Ginger supplementation also led to a significant decrease in body weight, body mass index, waist circumference, serum TSH, FBS, TG, and TChol levels compared to the placebo. In summary according to preliminary results of this study, ginger supplementation can help relieve persistent hypothyroid symptoms. Also, it may have beneficial effects in terms of weight reduction and regulation of the FBS and lipid profile in hypothyroid patients.

\section{Introduction}

Primary hypothyroidism is a clinical syndrome in which the thyroid gland does not produce enough thyroid hormones. The disease is classified as overt hypothyroidism and subclinical hypothyroidism $[1,2]$. Overt hypothyroidism is characterized by an increase in the serum level of thyroidstimulating hormone (TSH) and a decrease in the serum level of free thyroxine (T4), whereas the main feature of subclinical hypothyroidism is a normal free $\mathrm{T} 4$ 
concentration in the presence of an elevated serum TSH concentration $[3,4]$. The prevalence of hypothyroidism is generally $1-2 \%$, with five to eight times greater prevalence among women [5]. The most common symptoms and signs of hypothyroidism include fatigue, voice changes, cold intolerance, weight gain, constipation, dry skin, malaise, menstrual irregularities, decreased resting metabolism, dyslipidemia, and insulin resistance [6,7].

Replacement synthetic thyroxine (levothyroxine) is the standard therapy for hypothyroidism $[8,9]$. The goal of therapy is the normalization of the serum TSH level $[8,9]$. After thyroid hormone replacement therapy, approximately $15 \%$ of patients remain symptomatic despite achieving normal serum TSH levels $[6,10]$. The pathophysiology of persistent hypothyroid symptoms despite adequate hormone replacement therapy is not well understood $[11,12]$, and no definitive treatment is available $[13,14]$. Levothyroxine-liothyronine combination therapy is a suggested treatment for this group of patients with conflicting results $[13,15]$.

Zingiber officinale is a perennial plant from the Zingiberaceae family. Its rhizome (underground stem), commonly known as ginger, is widely used as a popular spice in different cultures around the world $[16,17]$. The Food and Drug Administration (FDA) recognizes ginger as a safe dietary supplement [18]. It also has long been used in traditional medicines as a hot remedy for the treatment of cold temperament symptoms and signs such as tiredness, constipation, obesity, and menstrual irregularities $[19,20]$. According to the similarities between the mentioned symptoms and hypothyroid symptoms, warm remedies such as ginger are recommended by traditional medicine practitioners for these patients [20]. Besides, several scientific investigations have confirmed the therapeutic effects of ginger on hyperlipidemia [21, 22], insulin resistance [23, 24], and obesity [22,25], which are all common comorbidities in hypothyroid patients. Recent studies also have shown the protective effect of ginger against thyroid damage in animals and humans $[16,26,27]$.

Based on the traditional use of ginger supplementation in the improvement of hypothyroidism-like symptoms as well as current evidence of the therapeutic effects of ginger on the comorbidities of hypothyroidism, the present study was designed to evaluate the efficacy of ginger supplementation in alleviating persistent hypothyroid symptoms and treating the related comorbidities among patients with adequate hormone replacement therapy.

\section{Materials and Methods}

2.1. Registration and Ethical Issues. The current study was registered in the Iranian Registry of Clinical Trials (IRCT; "this trial is registered with https://trialsearch.who.int/? TrialID=IRCT20191231045961N1"). All participants provided signed informed consent. The study protocol was approved by the Ethics Committee of Shiraz University of Medical Sciences (IR.SUMS.MED.REC.1398.548).
2.2. Study Design. This study was a single-center, doubleblind, placebo-controlled, randomized clinical trial with two parallel arms. No changes were made to the method after starting the trial.

2.3. Participants. Participants enrolled in this study were men and women aged 20-60 years with a previously confirmed diagnosis of overt primary hypothyroidism under hormone replacement therapy with normal serum TSH concentration $(0.3-4.5 \mathrm{mIU} / \mathrm{L})$ and a body mass index (BMI) of $19-35 \mathrm{~kg} / \mathrm{m}^{2}$ who were referred to Yasuj (south of Iran) endocrinology clinics between August and November 2020.

Exclusion criteria were pregnancy, lactation, alcohol consumption, diabetes mellitus, dyslipidemia, vitamin D deficiency, co-occurrence of other thyroid diseases, malignancy, liver and kidney failure, hypopituitarism, malabsorption disorders (celiac disease, inflammatory bowel disease, and atrophic gastritis), concomitant use of drugs such as multivitamins, minerals, phenobarbital, phenytoin, carbamazepine, rifampin, glucocorticoids, warfarin, statins, estrogen, and raloxifene, and history of hypersensitivity to ginger supplements.

2.4. Sample Size and Randomization. By applying data of previous studies [16] and using the MedCalc software with a $5 \%$ significance level and a power of $80 \%$, the sample size in each group was determined to be 30 cases. Based on the list from the random allocation software, thirty patients were placed in each group using the block randomization method with a block size of 4 .

2.5. Ginger and Placebo Capsules' Preparation. Ginger rhizomes were purchased from the local market and authenticated by a botanist from the Department of Phytopharmaceuticals, School of Pharmacy, Shiraz University of Medical Sciences, Shiraz, Iran, with a specified voucher number (PM -1142). Then, the ginger rhizomes were pulverized and exposed to ultraviolet light. Then, capsules were filled with $500 \mathrm{mg}$ of ginger powder. To prepare the placebo, the second group of capsules were filled with $500 \mathrm{mg}$ of roasted starch. To ensure odor matching, the placebo capsules (with the same appearance as ginger capsules) were placed in the presence of ginger powder for two weeks. Then, both groups of capsules were packed using the same packaging but were coded with different codes. All of these steps were performed in the Department of Traditional Pharmacy of the Faculty of Pharmacy of Shiraz University of Medical Sciences. To keep the study doubleblind, the codes were broken after recording the information of the last participant.

2.6. Intervention. Prior to commencing the intervention, the objectives of the study and the possible side effects of the drugs were explained to each patient, and written informed 
consent was obtained. Then, study questionnaires were filled out by the enrolled patients to assess their symptoms. Next, the patients were randomly assigned to the two study groups. Finally, according to previous studies [28-30], the participants received one $500 \mathrm{mg}$ capsule containing ginger powder in the intervention group and one starch-containing capsule in the placebo group $30 \mathrm{~min}$ before lunch and dinner (twice daily) for 30 days. All participants were on medication (levothyroxine $30 \mathrm{~min}$ before breakfast) and were advised not to change their levothyroxine dose, lifestyle, and physical activity during the study.

2.7. Outcome Measures. The primary outcome was the clinical symptoms as evaluated by the Thyroid Symptom Rating Questionnaire (ThySRQ), which is a validated disease-specific questionnaire for hypothyroidism [31]. The ThySRQ consists of 15 questions with a four-point symptom bother scale (not at all (0), a little (1), quite a bit (2), and very much (3)) that asks about the symptoms of hypothyroidism that patients may have experienced in recent weeks (3-6 weeks) and measures the perceived symptom severity. Secondary outcome measures were body weight, BMI, waist circumference, triglyceride (TG), total cholesterol (TChol), fasting blood sugar (FBS), and TSH, which were evaluated before and after the intervention. Blood samples were taken from all participants after 14 hours of overnight fasting. All patients were instructed to report any observed adverse events during the study.

2.8. Statistical Analysis. All statistical analyses were performed using the SPSS software (version 21.0; IBM Corp., Armonk, NY, USA). The normality of the data was examined using the Shapiro-Wilk test. The independent sample $t$-test and paired sample $t$-test were used to compare quantitative variables. The chi-squared test was used to compare qualitative variables in both groups. If the chi-square test was not suitable, Fisher's exact test was used. If the data distribution was not normal, the median and midrange were used to describe the data; such data were assessed using the Mann-Whitney $U$ test for intergroup comparison and the Wilcoxon test for intragroup comparison. Quantitative data were expressed as mean \pm standard deviation (SD), while qualitative variables were reported using frequency and percentage. In all tests, statistical significance was considered when the $P$ value was below 0.05 .

\section{Results}

3.1. Study Flow. In the course of the study (30 days), out of the 60 patients with a mean age of $40.47 \pm 8.35$ years and a mean weight of $83.45 \pm 16.93 \mathrm{~kg}$ who were enrolled, 7 patients were excluded because of coronavirus disease 2019 (COVID-19) affliction, poor compliance, or migration. Finally, 53 patients reached the data analysis stage, of which 27 patients were in the ginger group and 26 were in the placebo group. The details of patients' enrollment, follow-up, and analysis are demonstrated in Figure 1.
3.2. Baseline Demographic and Anthropometric Characteristics. The baseline demographic and anthropometric characteristics of the patients are shown in Table 1. Comparison of these characteristics shows that there was no significant difference in age, sex, BMI, and waist circumference between the study groups $(P>0.05)$.

3.3. Hypothyroid Symptoms. After the patients receiving ginger supplementation showed a significant decrease in the mean total ThySRQ score compared to the placebo group (Figure 2). Specifically, ginger led to significant changes in weight gain, cold feeling, constipation, dry skin, appetite, memory loss, concentration disturbance, and feeling giddy or dizzy domains, while the remaining domains showed no significant changes $(P>0.05)$. The details of information on the mean ThySRQ score before and after the intervention in both study groups are summarized in Table 2 .

3.4. Anthropometric Measures. Ginger supplementation led to a significant decrease in body weight, BMI, and waist circumference when compared to the placebo group. The anthropometric outcomes before and after the intervention are summarized in Table 3.

3.5. Laboratory Indices. Clinically minor but statistically significant improvements were also observed in the laboratory outcomes including the TSH, FBS, TG, and TChol levels in the ginger group compared to the control. The related data are presented in Table 3.

3.6. Physical Activity. The participants' level of physical activity according to the International Physical Activity Questionnaire (IPAQ) is reported in Table 3. The results indicate that there was no significant difference within and between the groups in terms of physical activity at the beginning and after the intervention.

3.7. Adverse Events. No patient in the ginger or placebo group reported any adverse event throughout the study period.

3.8. Gender-Based Subgroup Analysis. Among the total 53 patients included in the final analysis, 40 patients $(75.5 \%)$ were female. There was no statistically significant difference between the mean scores of ThySRQ domains and laboratory indices (TSH, FBS, TG, and TChol) in males and females receiving ginger $(P>0.05)$. The results of gender-based subgroup analysis are presented in Table 4.

\section{Discussion}

The present study results revealed that ginger supplementation in the dose of $1000 \mathrm{mg} / \mathrm{d}$ can alleviate persistent hypothyroid symptoms after adequate hormone replacement therapy. It also showed beneficial effects on certain anthropometric and laboratory outcomes in these patients. 


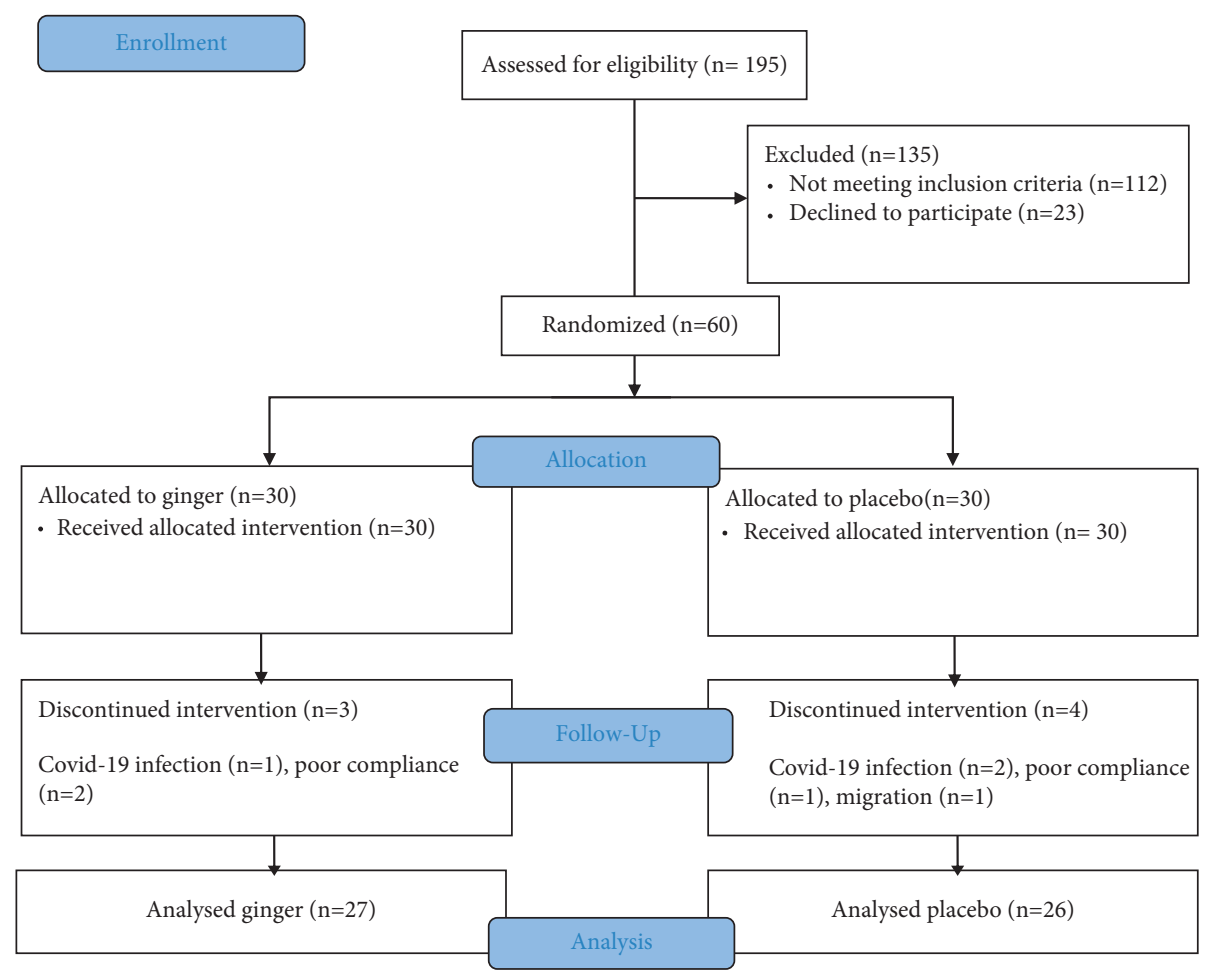

Figure 1: CONSORT flow diagram of the patients' enrollment and follow-up.

TABLE 1: Baseline demographic and anthropometric and laboratory characteristics of hypothyroid patients.

\begin{tabular}{lccc}
\hline Characteristics & Ginger $($ mean $\pm \mathrm{SD}), n=27$ & Placebo $(\mathrm{mean} \pm \mathrm{SD}), n=26$ & $P$ value \\
\hline Gender $($ male/female $)$ & $9(33 \%) / 18(67 \%)$ & $4(15 \%) / 22(85 \%)$ & 0.129 \\
Age $($ years $)$ & $42.44 \pm 8.48$ & $38.42 \pm 7.86$ & 0.08 \\
BMI $\left(\mathrm{kg} / \mathrm{m}^{2}\right)$ & $31.17 \pm 5.28$ & $31.29 \pm 5.12$ & 0.928 \\
Waist size $(\mathrm{cm})$ & $110.3 \pm 11.52$ & $109.5 \pm 13.92$ & 0.821 \\
TSH & $3.62 \pm 2.21$ & $2.87 \pm 1.88$ & 0.185 \\
IPAQ score & $200.36 \pm 95.69$ & $200.35 \pm 61.44$ & 1.000 \\
\hline
\end{tabular}

${ }^{\dagger} t$-test for quantitative variable; chi-square for qualitative variable. BMI: body mass index; TSH: thyroid-stimulating hormone; IPAQ: International Physical Activity Questionnaire.

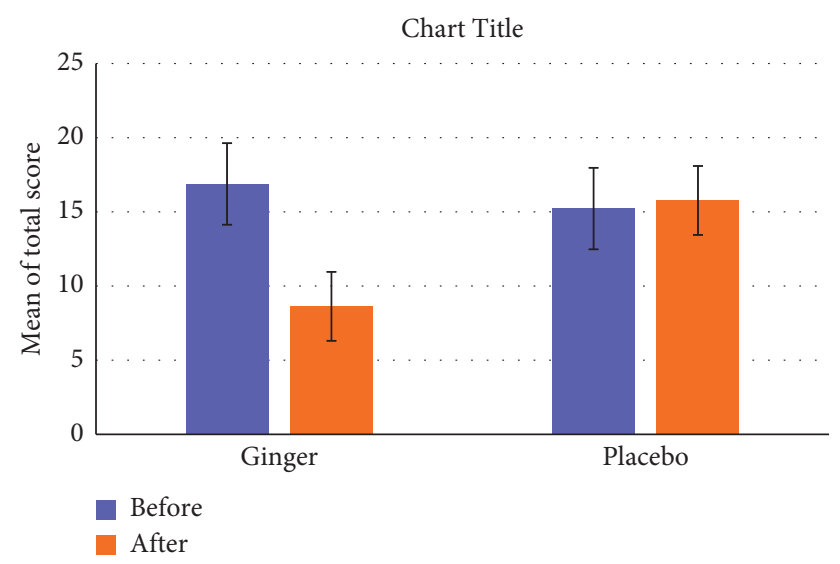

FIgURE 2: Change of total ThySRQ score between and within groups before and after the intervention. 
TABLE 2: Comparison of the changes in mean Hypothyroidism Symptom Rating Questionnaire (ThySRQ) scores between intervention groups.

\begin{tabular}{|c|c|c|c|c|}
\hline \multicolumn{2}{|c|}{ ThySRQ domains/intervention } & Ginger (mean \pm SD), $n=27$ & Placebo (mean \pm SD), $n=26$ & $P$ value* \\
\hline \multirow{3}{*}{ Tiredness } & Before & $2.19 \pm 0.89$ & $2.31 \pm 0.90$ & 0.501 \\
\hline & After & $0.73 \pm 0.72$ & $2.36 \pm 0.93$ & $<0.001$ \\
\hline & $p$ value ${ }^{* *}$ & $<0.001$ & 0.161 & \\
\hline \multirow{3}{*}{ Weight gain } & Before & $1.71 \pm 0.78$ & $1.42 \pm 0.90$ & 0.074 \\
\hline & After & $0.00 \pm 0.00$ & $1.11 \pm 0.85$ & $<0.001$ \\
\hline & $p$ value & $<0.001$ & 0.356 & \\
\hline \multirow{3}{*}{ Felt colder than other people } & Before & $1.54 \pm 0.98$ & $1.47 \pm 0.98$ & 0.729 \\
\hline & After & $0.02 \pm 0.14$ & $1.93 \pm 1.12$ & $<0.001$ \\
\hline & $p$ value & $<0.001$ & $<0.001$ & \\
\hline \multirow{3}{*}{ Constipation } & Before & $1.06 \pm 0.94$ & $0.98 \pm 0.97$ & 0.682 \\
\hline & After & $0.38 \pm 0.69$ & $1.04 \pm 1.05$ & $<0.001$ \\
\hline & $p$ value & 0.004 & 0.664 & \\
\hline \multirow{3}{*}{ Hair loss or coarseness } & Before & $1.63 \pm 1.19$ & $1.31 \pm 1.25$ & 0.170 \\
\hline & After & $1.40 \pm 1.19$ & $1.33 \pm 1.26$ & 0.748 \\
\hline & $p$ value & 0.017 & 0.327 & \\
\hline \multirow{3}{*}{ Skin dryness or coarseness } & Before & $1.13 \pm 1.03$ & $0.89 \pm 0.90$ & 0.194 \\
\hline & After & $1.62 \pm 1.16$ & $1.04 \pm 0.98$ & 0.006 \\
\hline & $p$ value & $<0.001$ & 0.011 & \\
\hline \multirow{3}{*}{ Nail brittleness or flaking } & Before & $0.40 \pm 0.69$ & $0.42 \pm 0.69$ & 0.915 \\
\hline & After & $0.40 \pm 0.69$ & $0.44 \pm 0.69$ & 0.808 \\
\hline & $p$ value & 0.999 & 0.999 & \\
\hline \multirow{3}{*}{ Loss of appetite } & Before & $0.13 \pm 0.44$ & $0.33 \pm 1.16$ & 0.263 \\
\hline & After & $0.00 \pm 0.00$ & $0.33 \pm 1.16$ & 0.044 \\
\hline & $p$ value & 0.056 & 1.000 & \\
\hline \multirow{3}{*}{ Hearing problems } & Before & $0.48 \pm 0.73$ & $0.22 \pm 0.57$ & 0.039 \\
\hline & After & $0.38 \pm 0.66$ & $0.22 \pm 0.57$ & 0.165 \\
\hline & $p$ value & 0.043 & 0.999 & \\
\hline \multirow{3}{*}{ Voice hoarseness or huskiness } & Before & $0.56 \pm 0.80$ & $0.60 \pm 0.83$ & 0.789 \\
\hline & After & $0.42 \pm 0.67$ & $0.58 \pm 0.85$ & 0.288 \\
\hline & $p$ value & 0.011 & 0.327 & \\
\hline \multirow{3}{*}{ Speech change (slowness, inaccuracy) } & Before & $0.56 \pm 1.51$ & $0.22 \pm 0.50$ & 0.118 \\
\hline & After & $0.31 \pm 0.64$ & $0.22 \pm 0.50$ & 0.421 \\
\hline & $p$ value & 0.282 & 0.999 & \\
\hline \multirow{3}{*}{ Memory problems } & Before & $1.63 \pm 0.93$ & $1.53 \pm 0.96$ & 0.558 \\
\hline & After & $0.75 \pm 0.76$ & $1.60 \pm 0.99$ & $<0.001$ \\
\hline & $p$ value & $<0.001$ & 0.161 & \\
\hline \multirow{3}{*}{ Concentration disturbance } & Before & $1.29 \pm 0.91$ & $1.07 \pm 0.81$ & 0.200 \\
\hline & After & $0.58 \pm 0.72$ & $1.05 \pm 0.85$ & 0.002 \\
\hline & $p$ value & $<0.001$ & 1.000 & \\
\hline \multirow{3}{*}{ Giddy or dizzy feeling } & Before & $0.81 \pm 0.72$ & $0.67 \pm 0.64$ & 0.305 \\
\hline & After & $0.10 \pm 0.41$ & $0.71 \pm 0.69$ & $<0.001$ \\
\hline & $p$ value & $<0.001$ & 0.574 & \\
\hline \multirow{3}{*}{ Depressed or low feeling } & Before & $1.75 \pm 0.99$ & $1.78 \pm 1.12$ & 0.877 \\
\hline & After & $1.54 \pm 1.06$ & $1.82 \pm 1.12$ & 0.188 \\
\hline & $p$ value & 0.376 & 0.574 & \\
\hline
\end{tabular}

ThySRQ: Thyroid Symptom Rating Questionnaire. ${ }^{*} p$ value Student's $t$-test. ${ }^{* *} p$ value paired $t$-test.

Hypothyroidism is a chronic disease with multiple physiological and psychological symptoms that require long-life treatment, exerting a remarkable negative effect on the patient's quality of life (QoL) [32]. Winther $\mathrm{KH}$ et al. demonstrated that treatment with levothyroxine for six weeks significantly improved the QoL of hypothyroidism patients compared to the general population [33]. Nevertheless, approximately $15 \%$ of patients remain symptomatic despite achieving normal serum TSH levels [34]. Symptoms of hypothyroidism include a wide range such as fatigue, cold intolerance, dry skin, hair loss, constipation, decreased appetite, memory loss, concentration disturbance, and feeling giddy or dizzy which are sometimes accompanied by other underlying conditions, including chronic depression, gastrointestinal problems, or anemia [3, 35]. Therefore, questionnaires which rate thyroid symptoms such as ThySRQ are nonspecific, and the recorded symptoms can be due to a wide range of diseases. 
TABLE 3: Comparison of changes in mean anthropometric and laboratory indices within and between intervention groups.

\begin{tabular}{|c|c|c|c|c|}
\hline Parameters & Subgroups & Ginger (mean $\pm \mathrm{SD}), n=27$ & Placebo (mean $\pm \mathrm{SD}), n=26$ & $p$ value independent $t$-test \\
\hline \multirow{4}{*}{ Weight $(\mathrm{kg})$} & Before & $85.15 \pm 17.95$ & $81.69 \pm 15.97$ & 0.463 \\
\hline & After & $82.72 \pm 18.07$ & $82.81 \pm 15.90$ & 0.986 \\
\hline & Change & $-2.43 \pm 1.23$ & $1.12 \pm 1.18$ & 0.001 \\
\hline & $p$ value paired $t$-test & 0.001 & 0.001 & \\
\hline \multirow{4}{*}{ BMI $\left(\mathrm{kg} / \mathrm{m}^{2}\right)$} & Before & $31.17 \pm 5.28$ & $31.29 \pm 5.12$ & 0.928 \\
\hline & After & $30.27 \pm 5.32$ & $31.71 \pm 4.99$ & 0.315 \\
\hline & Change & $-0.90 \pm 0.46$ & $0.42 \pm 0.43$ & 0.001 \\
\hline & $p$ value paired $t$-test & 0.001 & 0.001 & \\
\hline \multirow{4}{*}{ Waist size $(\mathrm{cm})$} & Before & $110.30 \pm 11.52$ & $109.5 \pm 13.92$ & 0.821 \\
\hline & After & $106.33 \pm 11.25$ & $109.19 \pm 11.41$ & 0.363 \\
\hline & Change & $-3.96 \pm 1.81$ & $-0.31 \pm 8.97$ & 0.043 \\
\hline & $p$ value paired $t$-test & 0.001 & 0.863 & \\
\hline \multirow{4}{*}{ TSH } & Before & $3.62 \pm 2.21$ & $2.87 \pm 1.88$ & 0.185 \\
\hline & After & $2.76 \pm 1.72$ & $3.81 \pm 1.95$ & 0.042 \\
\hline & Change & $-0.89 \pm 1.33$ & $0.95 \pm 1.45$ & 0.001 \\
\hline & $p$ value paired t-test & 0.001 & 0.003 & \\
\hline \multirow{4}{*}{ FBS } & Before & $99.74 \pm 13.56$ & $86.19 \pm 8.32$ & 0.001 \\
\hline & After & $88.52 \pm 10.41$ & $93.15 \pm 8.78$ & 0.086 \\
\hline & Change & $-11.65 \pm 6.55$ & $6.96 \pm 7.51$ & 0.001 \\
\hline & $p$ value paired t-test & 0.001 & 0.001 & \\
\hline \multirow{4}{*}{ TG } & Before & $229.52 \pm 106.5$ & $163.04 \pm 79.49$ & 0.013 \\
\hline & After & $175.03 \pm 80.28$ & $203.93 \pm 104.52$ & 0.266 \\
\hline & Change & $-56.59 \pm 67.57$ & $40.89 \pm 57.44$ & 0.001 \\
\hline & $p$ value paired t-test & 0.001 & 0.001 & \\
\hline \multirow{4}{*}{ TChol } & Before & $181.56 \pm 41.54$ & $170.88 \pm 37.11$ & 0.329 \\
\hline & After & $162.89 \pm 32.87$ & $181.27 \pm 37.21$ & 0.062 \\
\hline & Change & $-19.38 \pm 24.02$ & $10.38 \pm 16.66$ & 0.001 \\
\hline & $p$ value paired t-test & 0.001 & 0.004 & \\
\hline \multirow{3}{*}{ IPAQ } & Before & $200.36 \pm 95.69$ & $200.35 \pm 61.44$ & 1.000 \\
\hline & After & $200.36 \pm 95.28$ & $200.28 \pm 60.46$ & 0.997 \\
\hline & $p$ value paired $t$-test & 0.995 & 0.915 & \\
\hline
\end{tabular}

BMI: body mass index; TSH: thyroid-stimulating hormone; FBS: fasting blood sugar; TG: triglyceride; TChol: total cholesterol; IPAQ: International Physical Activity Questionnaire.

TABLE 4: The results of gender-based subgroup analysis showing changes in IPAQ domains and laboratory indices in males and females who received ginger.

\begin{tabular}{|c|c|c|c|}
\hline IPAQ domains and laboratory indices & Male & Female & $P$ value \\
\hline Tiredness & $-1.33 \pm 0.71$ & $-1.72 \pm 0.57$ & 0.137 \\
\hline Weight gain & $-1.67 \pm 0.87$ & $-1.72 \pm 0.83$ & 0.873 \\
\hline Felt colder than other people & $-1.89 \pm 0.93$ & $-1.72 \pm 0.96$ & 0.671 \\
\hline Constipation & $0.00 \pm 1.32$ & $-0.94 \pm 0.73$ & 0.053 \\
\hline Hair loss or coarseness & $-0.22 \pm 0.44$ & $-0.28 \pm 0.57$ & 0.801 \\
\hline Skin dryness or coarseness & $0.67 \pm 0.50$ & $0.44 \pm 0.51$ & 0.294 \\
\hline Nail brittleness or flaking & $0.00 \pm .00$ & $0.00 \pm .00$ & 0.99 \\
\hline Loss of appetite & $-0.11 \pm 0.33$ & $-0.28 \pm 0.67$ & 0.49 \\
\hline Hearing problems & $-0.11 \pm 0.33$ & $-0.17 \pm 0.38$ & 0.715 \\
\hline Voice hoarseness or huskiness & $0.00 \pm 0.00$ & $-0.33 \pm 0.49$ & 0.052 \\
\hline Speech change (slowness, inaccuracy) & $0.00 \pm 0.00$ & $-0.61 \pm 2.35$ & 0.448 \\
\hline Memory problems & $-0.67 \pm 0.50$ & $-1.11 \pm 0.58$ & 0.062 \\
\hline Concentration disturbance & $-0.67 \pm 0.50$ & $-0.94 \pm 0.42$ & 0.139 \\
\hline Giddy or dizzy feeling & $-0.78 \pm 0.67$ & $-0.94 \pm 0.64$ & 0.534 \\
\hline Depressed or low feeling & $0.11 \pm 0.33$ & $-0.22 \pm 0.73$ & 0.209 \\
\hline TSH & $-0.75 \pm 0.69$ & $-0.97 \pm 1.59$ & 0.702 \\
\hline FBS & $-14.33 \pm 3.94$ & $-10.24 \pm 7.28$ & 0.132 \\
\hline TG & $-51.44 \pm 48.36$ & $-59.31 \pm 77.08$ & 0.784 \\
\hline TChol & $-20.33 \pm 18.63$ & $-18.88 \pm 26.96$ & 0.887 \\
\hline
\end{tabular}

IPAQ: International Physical Activity Questionnaire; FBS: fasting blood sugar; TG: triglyceride; TChol: total cholesterol. Negative values show a decrease in the mean scores through the intervention. 
In our study, before the intervention, the most common persistent symptoms despite adequate thyroid hormone replacement were cold intolerance and tiredness according to ThySRQ, which is in concordance with the studies of Winther et al. [33] and McMillan et al. [31]. MacLean et al. also suggested that tiredness, weight gain, and feeling cold are the most common symptoms in euthyroid yet symptomatic patients [36]. There is currently no definitive treatment to control symptoms in symptomatic patients who are biochemically euthyroid [12]. In our study, some symptoms were ameliorated significantly in the ginger group compared with the placebo group according to ThySRQ. These symptoms included weight gain, cold intolerance, constipation, dry skin, decreased appetite, memory loss, concentration disturbance, and feeling giddy or dizzy.

There is limited human and animal evidence about the effects of ginger supplementation on thyroid gland function. Mohammed et al. demonstrated that ginger extract had an antioxidant and protective effect against bisphenol A-induced thyroid injury in male rats, acting through the nuclear factor-like 2 (Nrf2)/heme oxygenase-1 (HO-1) pathway [26]. Their findings are in accordance with the results of AlAmoudi et al. [27], which showed the ameliorative effect of the ginger extract on thyroid toxicity induced by lambdacyhalothrin in albino rats. In these studies, intragastric gavage of bisphenol A or lambda-cyhalothrin created a cascade of histological changes in the thyroid gland, which led to decreased serum levels of thyroid hormones and increased TSH concentration. Notably, the synchronic gavage of the ginger extract resulted in a protective effect against the histological changes and led to a significant increase in the serum levels of thyroid hormones together with a remarkable decrease in TSH concentration [26,27]. In a clinical trial recently published by Mahassni et al., a result similar to that of our study was observed about the effect of ginger supplementation on the TSH level in patients with hypothyroidism. However, this group of researchers administered a higher dose of ginger ( $8.3 \mathrm{~g}$ of ginger extract) for a shorter study duration (3 weeks) [16]. Another study performed by Barari et al. demonstrated that the consumption of $10 \mathrm{mg} / \mathrm{kg} /$ day ginger extract for six weeks had no significant effect on TSH concentration, contrasting with our findings [37]. However, that study included a healthy population, whereas all of our patients suffered from primary hypothyroidism.

Besides improved TSH, other laboratory indices including FBS, TG, and TChol also improved after ginger supplementation compared with the baseline and the placebo group. Previous studies have shown the antidiabetic and antilipidemic effects of the ginger extract in animals and humans $[38,39]$. A study conducted by $\mathrm{Al}$ Hroob et al. in 2018 showed that the daily consumption of $800 \mathrm{mg} / \mathrm{kg} /$ day ginger extract for 6 weeks significantly reduced the FBS concentration in rats with streptozotocin-induced diabetes [40]. Chukwudike et al. also demonstrated the dose-dependent antidiabetic effect of ginger aqueous extract in male Wistar rats [41]. Subbaiah and colleagues revealed that the daily administration of $50-250 \mathrm{mg} / \mathrm{kg}$ ginger extract significantly reduced the lipid (TG and TChol) levels in male albino Wister rats [42]. In concordance with their study, Akanfe et al. explained that gavage of $400-600 \mathrm{mg} / \mathrm{kg} /$ day of ginger extract for four weeks decreased the TG and TChol concentration significantly in female Wistar rats [43]. Human studies also support these results. Murad et al. indicated that the daily consumption of $5 \mathrm{~g}$ of ginger powder for 12 weeks can reduce the TChol concentration in the healthy population [21]. Besides, Mushtaq et al. showed a significant decrease in the TChol concentration after 30 days of treatment with $3 \mathrm{~g}$ of ginger powder in patients with hyperlipidemia [44]. El Gayar et al. also demonstrated similar results in patients with diabetes mellitus after the administration of $1.8 \mathrm{~g}$ of ginger powder for 8 weeks, which led to reduced FBS, TG, and TChol concentrations [45]. However, no study before our report has evaluated such effects of ginger among patients with hypothyroidism.

Improvement of anthropometric measures in hypothyroid patients receiving ginger supplementation was another finding of our study. Although daily consumption of $1000 \mathrm{mg}$ of ginger led to weight loss and body mass index, lack of measurement of daily calorie intake and potential nonreported concomitant use of weight reduction drugs by patients are confounding factors that can affect our results. Nevertheless, previous animal studies showed significant effects of ginger on obesity [46,47]. However, there is controversy about the beneficial effects of ginger on obesity in human studies $[48,49]$, which may be due to differences in participants, duration, and the dose and pharmaceutical form of ginger. Similar to our study, Attari et al. illustrated that the administration of $2 \mathrm{~g}$ of ginger for 12 weeks in obese women significantly reduced the BMI and waist circumference compared with the baseline and the placebo group [17]. Also, Murad et al. demonstrated that the consumption of $5 \mathrm{~g}$ of ginger powder for 3 months significantly decreased the body weight in hyperlipidemic patients [21]. In a study conducted by Mozaffari et al. on 88 patients with type 2 diabetes, it was shown that the daily consumption of $3 \mathrm{~g}$ of ginger powder for 8 weeks did not affect the BMI [50]. Similarly, Arablou et al. explained that the administration of $1600 \mathrm{mg}$ of ginger powder for 12 weeks in patients with type 2 diabetes had no effect on BMI and waist circumference [38]. Our results showed for the first time that ginger improved the anthropometric outcomes in patients with hypothyroidism.

The Food and Drug Administration (FDA) recognizes ginger as a safe dietary supplement [18]. Clinical studies have shown that consuming ginger up to 2 grams per day has the least toxicity to humans. The maximum dose of ginger is 6 grams per day [51]. No serious side effects of using this plant have been observed in the therapeutic dose range. In some studies, hypersensitivity reactions have been reported in the form of dermatitis [52]. In some cases, patients complained of dyspepsia and worsening of gastrointestinal symptoms [53]. In the present study, no serious side effects of ginger consumption were reported, which may be due to the low dose and short duration of consumption.

Besides the strength of using a prospective design in this clinical trial and comparing the use of the studied agent against a placebo, our study had some limitations. The small 
sample size, short duration of the intervention, use of only one dose of ginger, and lack of assessment of daily calorie intake were the most important limitations.

\section{Conclusion}

According to preliminary results of this study, it was demonstrated that the daily consumption of $1000 \mathrm{mg}$ of ginger powder in patients with primary hypothyroidism on adequate hormone replacement (biochemically euthyroid) led to a significant reduction in hypothyroid symptoms. The most responsive symptoms to ginger supplementation were weight gain, cold intolerance, constipation, dry skin, decreased appetite, memory loss, concentration disturbance, and feeling giddy or dizzy. Furthermore, anthropometric outcomes including weight, BMI, and waist circumference as well as laboratory parameters such as FBS, TG, and total cholesterol also improved with ginger supplementation. It can be concluded that ginger supplementation in hypothyroid patients can help control persistent symptoms, decrease weight, and regulate the FBS and lipid profile. However, further human studies with larger sample sizes, longer durations, different ginger doses, and a follow-up period after discontinuation of the supplement are recommended.

\section{Data Availability}

The data used to support the findings of this study are available on request from the corresponding author.

\section{Conflicts of Interest}

All authors have no conflicts of interest to declare.

\section{Acknowledgments}

The authors wish to thank the Research Consultation Center for their invaluable assistance in editing this manuscript. Our study was financially supported by the Shiraz University of Medical Sciences(grant no. 97-17708).

\section{References}

[1] J. L. Jameson, Harrison's Principles of Internal Medicine, McGraw-Hill Professional, New York, NY, USA, 2018.

[2] R. L. F. Cecil, L. Goldman, and A. I. Schafer, "Goldman's cecil medicine, expert consult premium edition--enhanced online features and print, single volume, 24: Goldman's Cecil medicine," Elsevier Health Sciences, vol. 1, 2012.

[3] J. P. Almandoz and H. Gharib, "Hypothyroidism: etiology, diagnosis, and management," Medical Clinics of North America, vol. 96, no. 2, pp. 203-221, 2012.

[4] M. P. J. Vanderpump, W. M. G. Tunbrldge, J. M. French et al., "The incidence of thyroid disorders in the community: a twenty-year follow-up of the Whickham Survey," Clinical Endocrinology, vol. 43, no. 1, pp. 55-68, 1995.

[5] G. J. Canaris, N. R. Manowitz, G. Mayor, and E. C. Ridgway, "The Colorado thyroid disease prevalence study," Archives of Internal Medicine, vol. 160, no. 4, pp. 526-534, 2000.
[6] M. Freeman, G. A. Adunlin, C. Mercadel, S. Danzi, and I. Klein, "Hypothyroid symptoms in levothyroxine-treated patients," Innovations in pharmacy, vol. 10, no. 3, p. 19, 2019.

[7] G. Allen and S. Safranek, Secondary Causes of Obesity, Clinical Inquiries (MU), Ann Arbor, MI, USA, 2011.

[8] J. Jonklaas, A. C. Bianco, A. J. Bauer et al., "Guidelines for the treatment of hypothyroidism: prepared by the american thyroid association task force on thyroid hormone replacement," Thyroid, vol. 24, no. 12, pp. 1670-1751, 2014.

[9] J. B. Rugge, C. Bougatsos, and R. Chou, "Screening and treatment of thyroid dysfunction: an evidence review for the U.S. Preventive services task force," Annals of Internal Medicine, vol. 162, no. 1, pp. 35-45, 2015.

[10] M. D. Ettleson and A. C. Bianco, "Individualized therapy for hypothyroidism: is T4 enough for everyone?" Journal of Clinical Endocrinology \& Metabolism, vol. 105, no. 9, pp. e3090-e3104, 2020.

[11] S. Razvi, S. Mrabeti, and M. Luster, "Managing symptoms in hypothyroid patients on adequate levothyroxine: a narrative review," Endocrine Connections, vol. 9, no. 11, pp. R241-R250, 2020.

[12] M. T. McDermott, "Persistent hypothyroid symptoms despite adequate thyroid hormone replacement," in Management of Patients with Pseudo-endocrine Disorders, pp. 301-316, Springer, Berlin, Germany, 2019.

[13] R. Madan and F. S. Celi, "Combination therapy for hypothyroidism: rationale, therapeutic goals, and design," Frontiers in Endocrinology, vol. 11, 2020.

[14] J. Jonklaas, "Risks and safety of combination therapy for hypothyroidism," Expert Review of Clinical Pharmacology, vol. 9, no. 8, pp. 1057-1067, 2016.

[15] J. Jonklaas, E. Tefera, and N. Shara, "Prescribing therapy for hypothyroidism: influence of physician characteristics," Thyroid, vol. 29, no. 1, pp. 44-52, 2019.

[16] S. H. Mahassni and O. A. Bukhari, "Beneficial effects of an aqueous ginger extract on the immune system cells and antibodies, hematology, and thyroid hormones in male smokers and non-smokers," Journal of Nutrition \& Intermediary Metabolism, vol. 15, pp. 10-17, 2019.

[17] V. Ebrahimzadeh Attari, S. Mahluji, M. Asghari Jafarabadi, and A. Ostadrahimi, "Effects of supplementation with ginger (Zingiber officinale Roscoe) on serum glucose, lipid profile and oxidative stress in obese women: a randomized, placebocontrolled clinical trial," Pharmaceutical Sciences, vol. 21, no. 4, pp. 184-191, 2015.

[18] K. Singletary, "Ginger," Nutrition Today, vol. 45, no. 4, pp. 171-183, 2010.

[19] M. Fatemi Tekieh, "The association of hypothyroidism symptoms with cold dystemperament )Su-e-Mizaj-e Barid," Journal of Islamic and Iranian Traditional Medicine, vol. 10, no. 2, pp. 125-136, 2019.

[20] S. Parvinroo, F. Naghibi, S. Zahediasl, M. Kamalinejad, and M. Sabetkasaei, "The effects of seeds with hot and cold temperaments on serum thyroid hormones, corticosterone and urine vanillylmandelic acid concentrations of healthy rats," Journal of Ethnopharmacology, vol. 156, pp. 216-221, 2014.

[21] S. Murad, K. Niaz, and H. Aslam, "Effects of ginger on LDL-C, total cholesterol and body weight," Clinical and Medical Biochemistry, vol. 4, no. 140, pp. 2471-2663, Article ID 1000140, 2018.

[22] S. Kausar, "Effect of apple cider vinegar on glycemic control, hyperlipidemia and control on body weight in type 2 diabetes patients," Health Science, vol. 8, no. 5, pp. 59-74, 2019. 
[23] S. Jafarnejad, S. A. Keshavarz, S. Mahbubi et al., "Effect of ginger ( Zingiber officinale) on blood glucose and lipid concentrations in diabetic and hyperlipidemic subjects: a meta-analysis of randomized controlled trials," Journal of Functional Foods, vol. 29, pp. 127-134, 2017.

[24] H. Gholinezhad, H. Bazyar, H. Rashidi, P. Salehi, M. H. Haghighi-zadeh, and A. Zare Javid, "Using ginger supplement in adjunct with non-surgical periodontal therapy improves metabolic and periodontal parameters in patients with type 2 diabetes mellitus and chronic periodontitis: a double-blind, placebo-controlled trial," Journal of Herbal Medicine, vol. 20, Article ID 100315, 2020.

[25] J. Wang, W. Ke, R. Bao, X. Hu, and F. Chen, "Beneficial effects of gingerZingiber officinale Roscoeon obesity and metabolic syndrome: a review," Annals of the New York Academy of Sciences, vol. 1398, no. 1, pp. 83-98, 2017.

[26] E. T. Mohammed, K. S. Hashem, A. E. Ahmed, M. T. Aly, L. Aleya, and M. M. Abdel-Daim, "Ginger extract ameliorates bisphenol A (BPA)-induced disruption in thyroid hormones synthesis and metabolism: involvement of Nrf-2/HO-1 pathway," The Science of the Total Environment, vol. 703, Article ID 134664, 2020.

[27] W. M. Al-Amoudi, "Toxic effects of Lambda-cyhalothrin, on the rat thyroid: involvement of oxidative stress and ameliorative effect of ginger extract," Toxicology reports, vol. 5, pp. 728-736, 2018.

[28] H. Tabibi, H. Imani, S. Atabak, I. Najafi, M. Hedayati, and L. Rahmani, "Effects of ginger on serum lipids and lipoproteins in peritoneal dialysis patients: a randomized controlled trial," Peritoneal Dialysis International: Journal of the International Society for Peritoneal Dialysis, vol. 36, no. 2, pp. 140-145, 2016.

[29] Y. Li, "Preventive and protective properties of Zingiber officinale (ginger) in diabetes mellitus, diabetic complications, and associated lipid and other metabolic disorders: a brief review," Evidence-based Complementary and Alternative Medicine, vol. 2012, Article ID 516870, 2012.

[30] C. P. Ezenwa, "Effect of ginger (zingiber officinale) consumption as nutritional management recipe on lipid profile and hepatosteatosis status of non-diabetic patients with nonalcholic fatty liver disease," IJIRAS, vol. 12, 2019.

[31] C. McMillan, C. Bradley, S. Razvi, and J. Weaver, "Evaluation of new measures of the impact of hypothyroidism on quality of life and symptoms: the ThyDQoL and ThySRQ," Value in Health, vol. 11, no. 2, pp. 285-294, 2008.

[32] T. Watt, P. Cramon, L. Hegedüs et al., "The thyroid-related quality of life measure ThyPRO has good responsiveness and ability to detect relevant treatment effects," Journal of Clinical Endocrinology \& Metabolism, vol. 99, no. 10, pp. 3708-3717, 2014.

[33] K. H. Winther, P. Cramon, T. Watt et al., "Disease-specific as well as generic quality of life is widely impacted in autoimmune hypothyroidism and improves during the first six months of levothyroxine therapy," PLoS One, vol. 11, no. 6, Article ID e0156925, 2016.

[34] P. d. S. Vigário, F. Vaisman, C. M. Coeli et al., "Inadequate levothyroxine replacement for primary hypothyroidism is associated with poor health-related quality of life-a Brazilian multicentre study," Endocrine, vol. 44, no. 2, pp. 434-440, 2013.

[35] J. R. Garber, R. H. Cobin, H. Gharib et al., "Clinical practice guidelines for hypothyroidism in adults: cosponsored by the American association of clinical endocrinologists and the
American thyroid association," Thyroid, vol. 22, no. 12, pp. 1200-1235, 2012.

[36] S. G. MacLean, Personal Constructs and Adjustment in Secondary Hypothyroidism, University of Hertfordshire, Hatfield, England, 2011.

[37] R. Barari and S. Shirali, "Endurance training and ginger supplement on TSH, T3, T4 and testosterone and cortisol hormone in obese men," Iran Journal of Basic Medical and Science, vol. 3, pp. 96-103, 2016.

[38] T. Arablou, N. Aryaeian, M. Valizadeh, F. Sharifi, A. Hosseini, and M. Djalali, "The effect of ginger consumption on glycemic status, lipid profile and some inflammatory markers in patients with type 2 diabetes mellitus," International Journal of Food Sciences and Nutrition, vol. 65, no. 4, pp. 515-520, 2014.

[39] E. Pagano, "Ginger (Zingiber Officinale Roscoe) as a nutraceutical: focus on the metabolic, analgesic, and antiinflammatory effects," Phytotherapy Research, vol. 64, 2020.

[40] A. M. Al Hroob, M. H. Abukhalil, R. D. Alghonmeen, and A. M. Mahmoud, "Ginger alleviates hyperglycemia-induced oxidative stress, inflammation and apoptosis and protects rats against diabetic nephropathy," Biomedicine \& Pharmacotherapy, vol. 106, pp. 381-389, 2018.

[41] A. M. M. Chukwudike and B. O. Mercy, "Dose-dependent antidiabetic and antiobesity potentials of aqueous extract of Zingiber officinale Linn (Ginger) rhizomes in experimental diabetic rats: need for precaution," EC Nutrition, vol. 14, pp. 468-473, 2019.

[42] G. V. Subbaiah, K Mallikarjuna, B Shanmugam, S Ravi, P. U Taj, and K. S Reddy, "Ginger treatment ameliorates alcohol-induced myocardial damage by suppression of hyperlipidemia and cardiac biomarkers in rats," Pharmacognosy Magazine, vol. 13, no. 1, p. S69, 2017.

[43] O. R. Akanfe, I. A. Komolafe, and A. A. Iyanda, "The effect of ginger (zingiber officinale) on diet induced hyperlipidemia and tissue histology in adult female wistar rats: a biochemical and histopathological study," Journal of Complementary and Alternative Medical Research, vol. 18, pp. 1-8, 2019.

[44] S. Mushtaq, A. Z. Bilal, and S. F. H. Shah, "Effect of dried ginger (zingiber officinale) on serum total cholesterol level in hyperlipidemic patients," Annals of King Edward Medical University, vol. 24, no. 4, 2018.

[45] M. H. El Gayar, M. M. M. Aboromia, N. A. Ibrahim, and M. H. Abdel Hafiz, "Effects of ginger powder supplementation on glycemic status and lipid profile in newly diagnosed obese patients with type 2 diabetes mellitus," Obesity Medicine, vol. 14, Article ID 100094, 2019.

[46] H.-J. Kim, B. Kim, E.-G. Mun, S.-Y. Jeong, and Y.-S. Cha, "The antioxidant activity of steamed ginger and its protective effects on obesity induced by high-fat diet in C57BL/6J mice," Nutrition research and practice, vol. 12, no. 6, p. 503, 2018.

[47] J. Wang, D. Li, P. Wang, X. Hu, and F. Chen, “Ginger prevents obesity through regulation of energy metabolism and activation of browning in high-fat diet-induced obese mice," The Journal of Nutritional Biochemistry, vol. 70, pp. 105-115, 2019.

[48] M. S. Macit, S. Sözlü, B. Kocaadam, and N. Acar-Tek, "Evaluation of ginger (zingiber officinale roscoe) on energy metabolism and obesity: systematic review and meta-analysis," Food Reviews International, vol. 35, no. 7, pp. 685-706, 2019.

[49] N. Maharlouei, R. Tabrizi, K. B. Lankarani et al., "The effects of ginger intake on weight loss and metabolic profiles among overweight and obese subjects: a systematic review and metaanalysis of randomized controlled trials," Critical Reviews in 
Food Science and Nutrition, vol. 59, no. 11, pp. 1753-1766, 2019.

[50] H. Mozaffari-Khosravi, B. Talaei, B.-A. Jalali, A. Najarzadeh, and M. R. Mozayan, "The effect of ginger powder supplementation on insulin resistance and glycemic indices in patients with type 2 diabetes: a randomized, double-blind, placebo-controlled trial," Complementary Therapies in Medicine, vol. 22, no. 1, pp. 9-16, 2014.

[51] T. P. Staff, PDR for Herbal medicines, JAMA Network, Chicago, IL, USA, 2004

[52] S. F. Rahman, "Influence of curcumin and ginger in primary dysmenorrhea: a review," International Journal of Applied Engineering Research, vol. 15, no. 7, pp. 634-638, 2020.

[53] A. Supriani, "The effect OF boiled ginger drink jahecang ON blood pressure reduction IN hypertension patients IN the hamlet OF baban arosbaya village arosbaya district bangkalan regency," International Conference of Kerta Cendekia Nursing Academy, vol. 1, 2019. 